\title{
General solution of the scattering equations
}

\author{
Louise Dolan $^{a}$ and Peter Goddard ${ }^{b}$ \\ ${ }^{a}$ Department of Physics, University of North Carolina, \\ Chapel Hill, NC 27599, U.S.A. \\ ${ }^{b}$ School of Natural Sciences, Institute for Advanced Study, \\ Princeton, NJ 08540, U.S.A. \\ E-mail: Idolan@physics.unc.edu, pgoddard@ias.edu
}

ABSTRACT: The scattering equations, originally introduced by Fairlie and Roberts in 1972 and more recently shown by Cachazo, He and Yuan to provide a kinematic basis for describing tree amplitudes for massless particles in arbitrary space-time dimension, have been reformulated in polynomial form. The scattering equations for $N$ particles are equivalent to $N-3$ polynomial equations $h_{m}=0,1 \leq m \leq N-3$, in $N-3$ variables, where $h_{m}$ has degree $m$ and is linear in the individual variables. Facilitated by this linearity, elimination theory is used to construct a single variable polynomial equation, $\Delta_{N}=0$, of degree $(N-3)$ ! determining the solutions. $\Delta_{N}$ is the sparse resultant of the system of polynomial scattering equations and it can be identified as the hyperdeterminant of a multidimensional matrix of border format within the terminology of Gel'fand, Kapranov and Zelevinsky. Macaulay's Unmixedness Theorem is used to show that the polynomials of the scattering equations constitute a regular sequence, enabling the Hilbert series of the variety determined by the scattering equations to be calculated, independently showing that they have $(N-3)$ ! solutions.

Keywords: Scattering Amplitudes, Field Theories in Higher Dimensions, Field Theories in Lower Dimensions

ArXiv ePrint: 1511.09441 


\section{Contents}

1 Introduction 1

2 Review of the cases $N=4,5$ and $6 \quad 5$

2.1 Solutions for $N=4$ and $N=5 \quad 6$

2.2 Solution for $N=6 \quad 7$

2.3 Direct demonstration that $\Delta_{6}$ is the required sextic 8

2.4 Determination of $x, y$ in terms of $u, v$ satisfying $\Delta_{6}=0$

3 Elimination theory for $N=\mathbf{7} \quad \mathbf{9}$

3.1 Construction of the 24th order polynomial $\Delta_{7}$ using elimination theory $\quad 9$

3.2 Direct demonstration that $\Delta_{7}$ is the required polynomial of degree $24 \quad 11$

4 Elimination theory for general $N \quad 12$

4.1 Construction of the $(N-3)$ !-th degree polynomial $\Delta_{N}$ using elimination theory 12

$\begin{array}{lll}4.2 & \text { Direct demonstration that } \Delta_{N} \text { is the required polynomial } & 13\end{array}$

$\begin{array}{ll}4.3 \text { The leading power of } v, v^{\delta_{N}} & 14\end{array}$

5 Resultants, hyperdeterminants and Hilbert series $\quad 17$

$\begin{array}{lll}5.1 & \text { Sparse resultants } & 17\end{array}$

$\begin{array}{lll}5.2 & \text { Hyperdeterminants and the scattering equations } & 18\end{array}$

$\begin{array}{lll}5.3 & \text { Hilbert series and regular sequences } & 20\end{array}$

\section{Introduction}

In this paper, we describe the solution of the scattering equations originally introduced by Fairlie and Roberts in 1972 [1-3] in a search for new dual models (string theories) without tachyons and subsequently found again by Gross and Mende while studying the high-energy behavior of string theory [4]. Recently they have been rediscovered by Cachazo, He and Yuan (CHY) [5]-[8], who have shown that they provide a basis for describing the kinematics of massless particles, by proposing remarkable formulae for their tree amplitudes, which have been proved for scalar field theory and gauge theory [9].

We consider $N$ massless particles, labelled by $a \in A=\{1,2, \ldots, N\}$, with momenta, $k_{a}, a \in A$, with $k_{a}^{2}=0$ and $\sum_{a \in A} k_{a}=0$, and introduce a variable $z_{a} \in \mathbb{C}$ for each $a \in A$. Then the scattering equations are the $N$ equations $f_{a}(z, k)=0, a \in A$, where

$$
f_{a}(z, k)=\sum_{\substack{b \in A \\ b \neq a}} \frac{k_{a} \cdot k_{b}}{z_{a}-z_{b}} .
$$


This system of $N$ equations is Möbius invariant system, and consequently satisfy three relations,

$$
\sum_{a \in A} f_{a}(z, k)=0 ; \quad \sum_{a \in A} z_{a} f_{a}(z, k)=0 ; \quad \sum_{a \in A} z_{a}^{2} f_{a}(z, k)=0,
$$

so that there are only $N-3$ linearly independent equations in the system.

The equations (1.2) can clearly be written in polynomial form by multiplying by suitable factors of $z_{a}-z_{b}$, but it is desirable to obtain a set of polynomials whose degrees are as small as possible. The equations typically have $(N-3)$ ! solutions [5], up to Möbius transformations, and so Bézout's theorem, which states that the number of solutions of a system of $M$ polynomial equations in $M$ unknowns is the product of the degrees of the polynomials, suggests that there should be a system of $N-3$, equations of degrees $1,2, \ldots N-3$, that is equivalent to the scattering equations, up to Möbius transformations, and this has been shown to be the case [10].

The proposals of CHY for tree amplitudes in massless theories were expressed as integrals of rational functions of the $z_{a}$ and the momenta, around poles occurring at the each of the solutions of the scattering equations. These integrals are hence just sums over the solutions and thus, necessarily, rational functions of the coefficients in the scattering equations. Therefore, the prescription is fundamentally one of attaching an algebraic expression to the scattering equations, or, equivalently, the zero-dimensional variety that they describe; the integral is somewhat symbolic. The objective is to understand the amplitudes in terms of natural algebraic objects attached to this variety.

A number of authors have discussed the use of techniques of algebraic geometry and commutative algebra to discuss the polynomial scattering equations [11]-[14]. There are also extensions of the scattering equations to massive particles [9, 15]; discussions of world sheet theories and loop amplitudes [16]-[19], leading to one-loop (or torus) scattering equations that can also be put in polynomial form [20-22]; and discussions of the loop amplitudes by mapping onto the Riemann sphere [23]-[27], but we shall not discuss any of these further here.

The scattering equations are equivalent [10] to the $N-3$ polynomial equations $\tilde{h}_{m}(z, k)=$ $0,2 \leq m \leq N-2$, where

$$
\tilde{h}_{m}(z, k)=\sum_{\substack{S \subset A \\|S|=m}} k_{S}^{2} z_{S}, \quad 2 \leq m \leq N-2,
$$

the sum is over all $N ! / m !(N-m) !$ subsets $S \subset A$ with $m$ elements, and

$$
k_{S}=\sum_{b \in S} k_{b}, \quad z_{S}=\prod_{a \in S} z_{a}, \quad S \subset A .
$$

This can be established by an argument closely related to the original motivation of Fairlie and Roberts for introducing the scattering equations. They replaced the Virasoro conditions of string theory by the condition

$$
p(z)^{2}=\sum_{a, b \in A} \frac{k_{a} \cdot k_{b}}{\left(z-z_{a}\right)\left(z-z_{b}\right)}=0,
$$


where

$$
p(z)^{\mu}=\sum_{a \in A} \frac{k_{a}^{\mu}}{z-z_{a}} .
$$

$p(z)^{2}$ has no double poles because $k_{a}^{2}=0, a \in A$, and, since it vanishes sufficiently fast at infinity, it will vanish everywhere provided it has no pole at $z=z_{a}$, for each $a \in A$, and this condition is just the scattering equations (1.1). However, the vanishing of $p(z)^{2}$ is equivalent to the vanishing of the polynomial of degree $N-2$,

$$
F(z)=p(z)^{2} \prod_{a \in A}\left(z-z_{a}\right)=\sum_{\substack{S \subset A \\|S|=2}} k_{S}^{2} \prod_{b \in \bar{S}}\left(z-z_{b}\right),
$$

where $\bar{S}=\{a \in A: a \notin S\}$. It is straightforward to show that the coefficients of $z^{N-2}$ and $z^{N-3}$ in $F(z)$ vanish, and that

$$
F(z)=\sum_{m=2}^{N-2}(-1)^{m} z^{N-m-2} \tilde{h}_{m} .
$$

establishing the equivalence of the polynomial form (1.3) to the original scattering equations (1.1).

We can fix the Möbius invariance of the system partially by taking two of the $z, z_{1}$ and $z_{N}$, say, to $\infty$ and 0 , respectively.

$$
h_{m}(z, k)=\lim _{z_{1} \rightarrow \infty} \frac{\tilde{h}_{m+1}}{z_{1}}=\sum_{\substack{S \subset A^{\prime} \\|S|=m}} \sigma_{S} z_{S}, \quad 1 \leq m \leq N-3,
$$

where $A^{\prime}=\{a \in A: a \neq 1, N\}, \sigma_{S}=k_{S_{1}}^{2}$ and $S_{1}=S \cup\{1\} . h_{m}$ is an homogeneous polynomial of degree $m$ in the variables $z_{2}, \ldots, z_{N-1}$, linear in each of them separately. The equations $h_{m}(z, k)=0,1 \leq m \leq N-3$, define a (presumably) zero-dimensional projective variety in $\mathbb{C P}^{N-3}$, a set consisting typically of

$$
\prod_{m=1}^{N-3} \operatorname{deg} h_{m}=(N-3) !
$$

points by Bézout's Theorem. Writing $u=z_{N-2}, v=z_{N-1}$, we seek to construct a polynomial equation of degree $\delta_{N}=(N-3)$ ! in $u / v$, whose roots determine the solutions of the scattering equations.

As we shall see, while the degree of the $h_{m}$ determine the degree of this polynomial and so the number of solutions, it is the linearity of the $h_{m}$ in each $z_{a}$ taken separately that facilitates the derivation of the degree $\delta_{N}$ polynomial in a relatively simple explicit form. As we shall review in section 2, this has already been done for $N \leq 6$ [10]. For $N=4$ and 5, it is immediate: for $N=4$, there is just one linear equation, $h_{1}=0$, which just relates $u$ and $v$ and so determines their ratio; for $N=5$, the two equations, $h_{1}=h_{2}=0$, provide two relations between $x, u, v$, where $x=z_{2}$, linear in $x$, which, when $x$ is eliminated between them, provide the desired quadratic equation for $u / v$. 
For $N=6$, writing $x=z_{2}, y=z_{3}$, the equations $h_{1}=h_{2}=h_{3}=0$, although linear in the individual $z_{a}$, now involve $x y$ as well as $x, y$, and so the elimination of $x$ and $y$ is more complicated, but can be achieved by a simple application of the elimination theory developed by Sylvester and Cayley in the 19th century [28]-[30]. We supplement $h_{1}=h_{2}=h_{3}=0$ with $x h_{1}=x h_{2}=x h_{3}=0$ to provide six linear relations between $1, x, y, x y, x^{2}$ and $x^{2} y$, and the condition of their consistency is that the vanishing of the determinant of the matrix of their coefficients provides the desired sextic in $u / v$, which, as we review in section 2 , we can write the form $\Delta_{6}=0$ [10], where

$$
\Delta_{6}=\left|\begin{array}{cccccc}
h_{1} & h_{1}^{y} & h_{1}^{x} & h_{1}^{x y} & 0 & 0 \\
h_{2} & h_{2}^{y} & h_{2}^{x} & h_{2}^{x y} & 0 & 0 \\
h_{3} & h_{3}^{y} & h_{3}^{x} & h_{3}^{x y} & 0 & 0 \\
0 & 0 & h_{1} & h_{1}^{y} & h_{1}^{x} & h_{1}^{x y} \\
0 & 0 & h_{2} & h_{2}^{y} & h_{2}^{x} & h_{2}^{x y} \\
0 & 0 & h_{3} & h_{3}^{y} & h_{3}^{x} & h_{3}^{x y}
\end{array}\right|,
$$

with $h_{m}^{x}=\partial_{x} h_{m}, h_{m}^{x}=\partial_{x} \partial_{y} h_{m}$, etc. The advantage of this form is that we can see directly that $\Delta_{6}=0$ provides a sextic in $u / v$, vanishing when $h_{1}=h_{2}=h_{3}=0$, and so determining the solutions of the scattering equations, without relying on its derivation using elimination theory.

The sextic $\Delta_{6}$ is the resultant of $h_{1}, h_{2}, h_{3}$, regarded as polynomials in $x, y$. The resultant is the polynomial of lowest degree, with integral coefficients, whose variables are the coefficients of $h_{1}, h_{2}, h_{3}$, (which coefficients are themselves in this case homogeneous polynomials in $u, v$, at most linear in either) and which vanishes when the corresponding three equations have a common solution for $x, y$. As we discuss in section 5 , from elimination theory, we know that the resultant is irreducible (that is has no factors other than \pm 1 ) and is unique up to sign.

We shall see in section 3 that the discussion for $N=6$ extends to $N=7$, where we seek a polynomial equation of degree $\delta_{7}=24$, ideally obtained as the determinantal condition for the consistency of 24 independent linear relations between 24 monomials in $x, y, z$, where $x=z_{2}, y=z_{3}, z=z_{4}$, the equations being obtained by multiplying the polynomial scattering equations $h_{m}, 1 \leq m \leq 4$, by suitable monomials.

The general approach of elimination theory, as outlined in Cayley's classic article of 1848, which prefigured much of the development of commutative algebra [31], is to obtain new equations by multiplying the polynomial equations to be solved, treating these equations as linear relations between monomials, until the equations are overdetermined and so yield a consistency condition. It may be that the polynomial equations are not linearly independent and Cayley describes how to take account of the corresponding linear relations between the equations, and, potentially linear relations between these relations and so on. Of course, it is best if such complications can be avoided and fortunately we are able to do this for the polynomial scattering equations.

For $N=7$, we use the 24 equations given by

$$
h_{m}=y h_{m}=x h_{m}=x y h_{m}=x^{2} h_{m}=x^{2} y h_{m}=0, \quad 1 \leq m \leq 4,
$$


which provide linear relations between the 24 monomials

$$
x^{p} y^{q} z^{r}, \quad 0 \leq p \leq 3,0 \leq q \leq 2,0 \leq r \leq 1
$$

compromising 23 variables together with 1 . The determinantal condition for consistency of the equations (1.12), which we can show are linearly independent, provides the desired polynomial equation of degree 24 for $u / v$, which we can write in a form like (1.11).

As we show in section 4 , these results extend to general $N$. Writing $x_{a}=z_{a+1}, 1 \leq$ $a \leq N-4$, we consider the $(N-3)$ ! equations, $\alpha h_{m}=0,1 \leq m \leq N-3, \alpha \in C_{N-5}$, where $C_{M}$ denotes the set of monomials,

$$
C_{M}=\left\{\prod_{a=1}^{M} x_{a}^{m_{a}}: 0 \leq m_{a} \leq M-a+1,1 \leq a \leq M\right\} .
$$

These provide linear relations between the $(N-3)$ ! monomials $\beta \in C_{N-4}$, including unity. The resulting determinantal consistency condition is the required polynomial, $\Delta_{N}$, of order $\delta_{N}$ in $u / v$. Each element of the determinant can be taken to be a partial derivative of one of the $h_{m}$ as in (1.11); this is specified in (4.9).

Because $h_{m}$ is linear in each of the $z_{a}, 2 \leq a \leq N-1$, it is not a general sum of monomials in the $z_{a}$, and, for this reason it is not the theory of resultants for general multinomials that is relevant to the solution of the scattering equations but, as we outline in section 5 , the theory of sparse resultants. Further, the sparse resultant of multilinear equations of the form of the scattering equations is given by the hyperdeterminant of a multidimensional array, within the theory developed by Gel'fand, Kapranov and Zelevinsky [31]. Multilinear systems of this sort occur, e.g. in discussions of Nash equilibria in game theory (see chapter 6 of [32]); hyperdeterminants have previously occurred in theoretical physics, e.g. in the context of black hole entropy [33].

Information about the number of solutions of the scattering equations is encoded in the Hilbert series of the variety they determine. We calculate the Hilbert series by using Macaulay's Unmixedness Theorem [34] to show that the polynomials of the scattering equations constitute a regular sequence, and this provides an independent determination that they have $(N-3)$ ! solutions.

Some of our resultants overlap with work of Cardona and Kalousios [35], who also obtained the formula for the polynomial of order $\delta_{N}$, using an approach based on elimination theory.

\section{Review of the cases $N=4,5$ and 6}

As described in section 1 , in order to solve the scattering equations $f_{a}(z, k)=0, a \in A$, or, equivalently, $h_{m}(z, k)=0,1 \leq m \leq N-3$, we seek to eliminate $z_{a}, 2 \leq a \leq N-3$, in favor of $u=z_{N-2}$ and $v=z_{N-1}$; to give a polynomial of order $(N-3)$ ! in $u / v$. As before, when appropriate, write $x=z_{2}$ and $y=z_{3}$. 


\subsection{Solutions for $N=4$ and $N=5$}

For $N=4$, we have one simple linear equation determining $u / v$,

$$
h_{1}=\sigma_{2} u+\sigma_{3} v=0, \quad u / v=-\sigma_{3} / \sigma_{2}=-k_{1} \cdot k_{3} / k_{1} \cdot k_{2} .
$$

For $N=5$, eliminating $x$ between the equations

$$
\begin{aligned}
& h_{1}=\sigma_{2} x+\sigma_{3} u+\sigma_{4} v=0 \\
& h_{2}=\sigma_{23} x u+\sigma_{24} x v+\sigma_{34} u v=0
\end{aligned}
$$

yields a quadratic equation for $u / v$ given by the vanishing of the determinant

$$
\left|\begin{array}{cc}
\sigma_{3} u+\sigma_{4} v & \sigma_{2} \\
\sigma_{34} u v & \sigma_{23} u+\sigma_{24} v
\end{array}\right|=\left|\begin{array}{cc}
h_{1} & h_{1}^{x} \\
h_{2} & h_{2}^{x}
\end{array}\right|,
$$

where $h_{m}^{x}=\partial_{x} h_{m}$, as we see by adding $x$ times the second column to the first. Now, we can see directly that $\Delta_{5}=0$, where

$$
\Delta_{5}=\left|\begin{array}{ll}
h_{1} & h_{1}^{x} \\
h_{2} & h_{2}^{x}
\end{array}\right|,
$$

gives the required quadratic in $u / v$ because (i) it vanishes when $h_{1}=h_{2}=0$; (ii) it is a homogeneous quadratic in $(x, u, v)$; (iii) it is independent of $x$. To see that $\Delta_{5}$ is independent of $x$, note that

$$
\partial_{x} \Delta_{5}=\left|\begin{array}{ll}
h_{1}^{x} & h_{1}^{x} \\
h_{2}^{x} & h_{2}^{x}
\end{array}\right|+\left|\begin{array}{ll}
h_{1} & h_{1}^{x x} \\
h_{2} & h_{2}^{x x}
\end{array}\right|=0,
$$

since $h_{m}^{x x}=\partial_{x} h_{m}^{x}=0$, because $h_{m}$ is linear in $x$. To see that $\Delta_{5}$ is quadratic, note that the entry in row $r$ and column $c$ is of degree $r-c+1$; since the sum of the row numbers equals the sum of the column numbers for any product contributing to the determinant, $\Delta$ is of degree 2 . Note that the term in $v^{2}$ comes from the product $h_{1} h_{2}^{x}$ and its coefficient is $\sigma_{4} \sigma_{24}$.

Another way, which will be useful when we consider larger values of $N$, to establish the elementary fact that $\Delta_{5}$ is independent of $x$ is as follows. We are seeking the condition on $u, v$ (and the coefficients in the $h_{m}$ ) that $h_{1}(x, u, v)=0$ and $h_{2}(x, u, v)=0$ have a common solution for some value of $x$. For any given value of $x_{0}$, this is clearly the same as the condition that $h_{1}\left(x_{0}+\xi, u, v\right)=0$ and $h_{2}\left(x_{0}+\xi, u, v\right)=0$ have a common solution for some value of $\xi$. So this condition must be independent of $x_{0}$. But

$$
h_{m}\left(x_{0}+\xi, u, v\right)=h_{m}\left(x_{0}, u, v\right)+h_{m}^{x}\left(x_{0}, u, v\right) \xi, \quad m=1,2,
$$

and the condition for these equations to have a common solution for $\xi$ is $\Delta_{5}=0$ evaluated at $(x, u, v)=\left(x_{0}, u, v\right)$. So this condition is independent of $x_{0}$, which implies that $\Delta_{5}$, as defined by (2.4), is independent of $x$. 
Since $h_{1}, h_{2}$ and $h_{2}^{x}$ are linear in $v$ and $h_{1}^{x}$ is constant, the leading power of $v$ in $\Delta_{5}$, $v^{2}$, comes from the product $h_{1} h_{2}^{x}$ and its coefficient is

$$
\sigma_{4} \sigma_{24}
$$

The corresponding result for $N=4$ is that the coefficient of $v$ in $\Delta_{4}=h_{1}$ is $\sigma_{3}$. These leading coefficients are nonzero if all the Mandelstam variables $k_{S}^{2}, S \subset A$, are nonzero.

\subsection{Solution for $N=6$}

For $N=6$, we seek to eliminate $x$ and $y$ between the equations

$$
\begin{aligned}
& h_{1}=\sigma_{2} x+\sigma_{3} y+\sigma_{4} u+\sigma_{5} v=0, \\
& h_{2}=\sigma_{23} x y+\sigma_{24} x u+\sigma_{25} x v+\sigma_{34} y u+\sigma_{35} y v+\sigma_{45} u v=0, \\
& h_{3}=\sigma_{234} x y u+\sigma_{235} x y v+\sigma_{245} x u v+\sigma_{345} y u v=0,
\end{aligned}
$$

to obtain a sextic in $u, v$. We shall see that obtaining a simple formula for the polynomial in $u, v$ depends on each $h_{m}$ being linear in each $z_{a}$, while the degree of this polynomial depends on the degree of $h_{m}$ equalling $m$. We can write

$$
h_{m}=a_{m}+b_{m} y+c_{m} x+d_{m} x y, \quad m=1,2,3,
$$

where in fact $d_{1}=a_{3}=0$. Following the approach of elimination theory, we consider the equations, $h_{m}=0,1 \leq m \leq 3$, together with the equations $x h_{m}=0,1 \leq m \leq 3$, which provide six homogenous linear equations relating the five quantities $y, x, x y, x^{2}, x^{2} y$, the consistency of which leads to the condition $\Delta_{6}=0$, where

$$
\Delta_{6}=\left|\begin{array}{cccccc}
a_{1} & b_{1} & c_{1} & d_{1} & 0 & 0 \\
a_{2} & b_{2} & c_{2} & d_{2} & 0 & 0 \\
a_{3} & b_{3} & c_{3} & d_{3} & 0 & 0 \\
0 & 0 & a_{1} & b_{1} & c_{1} & d_{1} \\
0 & 0 & a_{2} & b_{2} & c_{2} & d_{2} \\
0 & 0 & a_{3} & b_{3} & c_{3} & d_{3}
\end{array}\right| .
$$

By row and column operations, we can show that we can rewrite $\Delta_{6}$ as

$$
\Delta_{6}=\left|\begin{array}{cccccc}
h_{1} & h_{1}^{y} & h_{1}^{x} & h_{1}^{x y} & 0 & 0 \\
h_{2} & h_{2}^{y} & h_{2}^{x} & h_{2}^{x y} & 0 & 0 \\
h_{3} & h_{3}^{y} & h_{3}^{x} & h_{3}^{x y} & 0 & 0 \\
0 & 0 & h_{1} & h_{1}^{y} & h_{1}^{x} & h_{1}^{x y} \\
0 & 0 & h_{2} & h_{2}^{y} & h_{2}^{x} & h_{2}^{x y} \\
0 & 0 & h_{3} & h_{3}^{y} & h_{3}^{x} & h_{3}^{x y}
\end{array}\right|,
$$

where $h_{m}^{x y}=\partial_{x} \partial_{y} h_{m}$. We can establish the condition $\Delta_{6}=0$ in the form (2.11), following the approach we used for $N=5$, by considering the condition for $h_{m}(x+\xi, y+\eta, u, v)=$ $0,1 \leq m \leq 3$, to have a common solution for $\xi, \eta$, given values of $x, y, u, v$, noting that this condition is clearly independent of the values of $x, y$. Since

$$
h_{m}(x+\xi, y+\eta, u, v)=h_{m}+h_{m}^{y} \eta+h_{m}^{x} \xi+h_{m}^{x y} \xi \eta, \quad m=1,2,3,
$$

where $h_{m}=h_{m}(x, y, u, v)$, etc., giving the vanishing of (2.11) as the condition. 


\subsection{Direct demonstration that $\Delta_{6}$ is the required sextic}

Again, we can see directly, without using elimination theory, that $\Delta_{6}$, as defined by (2.11), gives the required sextic in $u / v$ because (i) it vanishes when $h_{1}=h_{2}=h_{3}=0$; (ii) it is a homogeneous sextic in $(x, y, u, v)$; (iii) it is independent of $x, y$.

To see that $\Delta_{6}$ is a homogeneous sextic, note again that the entry in row $r$ and column $c$, if non-zero, is of degree $m_{r}-n_{c}+1$ in $x, y, u, v$, where $\left(m_{1}, m_{2}, m_{3}, m_{4}, m_{5}, m_{6}\right)=$ $(1,2,3,2,3,4)$ and $\left(n_{1}, n_{2}, n_{3}, n_{4}, n_{5}, n_{6}\right)=(1,2,2,3,3,4)$. Since any product contributing to the determinant involves one element from each row and one from each column, the degree of the product is 6 plus the sum of the $m_{r}$ less the sum of $n_{c}$, i.e. it equals 6 , showing that $\Delta_{6}$ is a homogeneous sextic. Because no element in the representation (2.11) is more than linear in $v$, the term in $v^{6}$ must be the product of linear factors, and it is then easy to see that it comes only from the product $h_{1} h_{2}^{y} h_{3}^{x y} h_{1} h_{2}^{x} h_{3}^{x y}$ and its coefficient is

$$
\sigma_{5}^{2} \sigma_{25} \sigma_{35} \sigma_{235}^{2}
$$

It is immediately obvious that $\partial_{y} \Delta_{6}=0$, because the $y$-derivative of any column either vanishes by linearity of the entries in $y$, or equals some other column. To show directly that $\partial_{x} \Delta_{6}=0$ in a way that will generalize to $N>6$, first write

$$
h^{\alpha}=\left(\begin{array}{c}
h_{1}^{\alpha} \\
h_{2}^{\alpha} \\
h_{3}^{\alpha}
\end{array}\right)
$$

where $\alpha=\varnothing, x, y$, or $x y$, with $h_{m}^{\varnothing} \equiv h_{m}$. Then

$$
\Delta_{6}=\left|\begin{array}{cccccc}
h & h^{y} & h^{x} & h^{x y} & 0 & 0 \\
0 & 0 & h & h^{y} & h^{x} & h^{x y}
\end{array}\right|,
$$

and, subtracting $x$ times column 6 from column 4 , and $x$ times column 5 from column 3 , and then $x$ times column 4 from column 2 , and $x$ times column 3 from column 1 ,

$$
\Delta_{6}=\left|\begin{array}{cccccc}
h_{o} & h_{o}^{y} & h^{x} & h^{x y} & 0 & 0 \\
-x h_{o} & -x h_{o}^{y} & h_{o} & h_{o}^{y} & h^{x} & h^{x y}
\end{array}\right|,
$$

where $h_{o}=h-x h^{x}$ and $h_{o}^{y}=h^{y}-x h^{x y}$; then adding $x$ times row 1 to row 2 , and then, again, subtracting $x$ times column 6 from column 4 , and $x$ times column 5 from column 3 , we obtain

$$
\Delta_{6}=\left|\begin{array}{cccccc}
h_{o} & h_{o}^{y} & h^{x} & h^{x y} & 0 & 0 \\
0 & 0 & h_{o} & h_{o}^{y} & h^{x} & h^{x y}
\end{array}\right|,
$$

which is manifestly independent of $x$.

\subsection{Determination of $x, y$ in terms of $u, v$ satisfying $\Delta_{6}=0$}

Given $u, v$ satisfying $\Delta_{6}=0, x, y$ can be determined from linear relations. Consider

$$
\Gamma_{6}=\left|h h^{x} h^{x y}\right|
$$


then $\partial_{x} \Gamma_{6}=0$ and

$$
\partial_{y} \Gamma_{6}=\left|h^{y} h^{x} h^{x y}\right|, \quad \partial_{y}^{2} \Gamma_{6}=0 .
$$

Thus $\Gamma_{6}$ is independent of $x$, linear in $y$ and vanishes when $h_{m}=0,1 \leq m \leq 3$, and so provides a linear relation to determine $y$, given $u, v$ satisfying $\Delta_{6}=0$. Similarly, $\left|h h^{y} h^{x y}\right|=0$ provides a linear relation, independent of $y$, to determine $x$.

We can derive the expression (2.18) for $\Gamma_{6}$ as follows. Given $u, v$ satisfying $\Delta_{6}=0$, and any $x, y$, we can find $\xi, \eta$ such that $h(x+\xi, y+\eta, u, v)=0$, implying $\Delta_{6}=0$. Then $\left(1, \eta, \xi, \xi \eta, \xi^{2}, \xi^{2} \eta\right)^{T}$ is a null vector of the matrix obtained by putting $h=h(x, y, u, v)$ in the matrix corresponding to the right hand side of (2.11) and

$$
\begin{aligned}
\eta=\frac{\xi \eta}{\xi} & =-\left|\begin{array}{ccccc}
h_{2} & h_{2}^{y} & h_{2}^{x} & 0 & 0 \\
h_{3} & h_{3}^{y} & h_{3}^{x} & 0 & 0 \\
0 & 0 & h_{1} & h_{1}^{x} & h_{1}^{x y} \\
0 & 0 & h_{2} & h_{2}^{x} & h_{2}^{x y} \\
0 & 0 & h_{3} & h_{3}^{x} & h_{3}^{x y}
\end{array}\right|\left|\begin{array}{ccccc}
h_{2} & h_{2}^{y} & h_{2}^{x y} & 0 & 0 \\
h_{3} & h_{3}^{y} & h_{3}^{x y} & 0 & 0 \\
0 & 0 & h_{1}^{y} & h_{1}^{x} & h_{1}^{x y} \\
0 & 0 & h_{2}^{y} & h_{2}^{x} & h_{2}^{x y} \\
0 & 0 & h_{3}^{y} & h_{3}^{x} & h_{3}^{x y}
\end{array}\right|^{-1} \\
& =-\left|\begin{array}{lll}
h_{1} & h_{1}^{x} & h_{1}^{x y} \\
h_{2} & h_{2}^{x} & h_{2}^{x y} \\
h_{3} & h_{3}^{x} & h_{3}^{x y}
\end{array}\right|\left|\begin{array}{ccc}
h_{1}^{y} & h_{1}^{x} & h_{1}^{x y} \\
h_{2}^{y} & h_{2}^{x} & h_{2}^{x y} \\
h_{3}^{y} & h_{3}^{x} & h_{3}^{x y}
\end{array}\right| .
\end{aligned}
$$

But, if $x, y$ are such that $h(x, y, u, v)=0, \eta=0$ and $\left|h h^{x} h^{x y}\right|=0$ determines $y$ given $u, v$ satisfying $\Delta_{6}=0$.

\section{Elimination theory for $N=7$}

\subsection{Construction of the 24th order polynomial $\Delta_{7}$ using elimination theory}

Writing $\left(z_{2}, z_{3}, z_{4}, z_{5}, z_{6}\right)=(x, y, z, u, v)$, we seek to eliminate $x, y$ and $z$ between the equations $h_{m}=0,1 \leq m \leq 4$, to obtain a homogeneous polynomial of order 24 in $u, v$. We can write

$$
h_{m}=a_{m}+b_{m} z+c_{m} y+d_{m} x+e_{m} y z+f_{m} x z+g_{m} x y+j_{m} x y z, \quad 1 \leq m \leq 4,
$$

and the column vector $h=\left(h_{1}, h_{2}, h_{3}, h_{4}\right)^{T}$; then $h=0$ provides 4 linear relations between 7 variables $x, y, z, x y, z x, y z, x y z$. Adding the 4 equations $x h=0$ brings in the additional variables $x^{2}, x^{2} y, z x^{2}, x^{2} y z$; further adding $y h=0$ brings in the variables $y, x y^{2}, y^{2} z, x y^{2} z$, giving a total of 15 variables with 12 linear relations, still leaving 3 more variables than relations. Then adding the 4 equations $x y h=0$, just adds two more variables $x^{2} y^{2}, x^{2} y^{2} z$, leaving one more variable than linear relation. Adding $x^{2} h=0$ leaves this balance unchanged with 4 more variables, $x^{3}, x^{3} y, z x^{3}, x^{3} y z$, to match the 4 new relations. But, finally, adding the 4 relations $x^{2} y h=0$ adds just the two variables $x^{3} y^{2}, x^{3} y^{2} z$, so giving a total of 23 variables with 24 linear relations, and so a consistency condition $\Delta_{7}=0$, where 
$\Delta_{7}$ is the 24 dimensional determinant

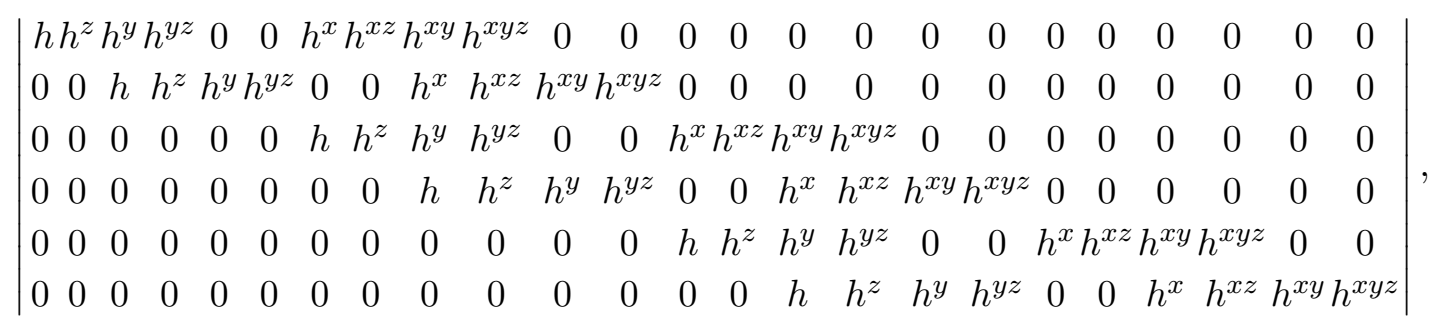

where $h_{m}^{x y z}=\partial_{x} \partial_{y} \partial_{z} h_{m}$. In principle, $h_{m}$ and its derivatives should be evaluated at $x=y=$ $z=0$, but we need not impose this condition because, as in the $N=5,6$ cases, for general $x, y, z$, the vanishing of (3.2) provides the condition that $h\left(x+x^{\prime}, y+y^{\prime}, z+z^{\prime}, u, v\right)=0$ has solutions for some values of $x^{\prime}, y^{\prime}, z^{\prime}$, and this is independent of $x, y, z$, and there is no need to impose the conditions $x=y=z=0$.

In (3.2), the rows correspond to $h, y h, x h, x y h, x^{2} h$ and $x^{2} y h$, respectively, and the columns to $1, z, y, y z, y^{2}, y^{2} z, x, x z, x y, x y z, x y^{2}, x y^{2} z, x^{2}, x^{2} z, x^{2} y, x^{2} y z, x^{2} y^{2}, x^{2} y^{2} z, x^{3}$, $x^{3} z, x^{3} y, x^{3} y z, x^{3} y^{2}$ and $x^{3} y^{2} z$, respectively. Defining $C_{N}$ as in (1.14), we can label the rows of (3.2) by $(\alpha, i), \alpha \in C_{2}, 1 \leq i \leq 4$, and its columns by $\beta \in C_{3}$. [Note $\left|C_{2}\right|=6,\left|C_{3}\right|=24$.] Then, if $B_{3}=\left\{x^{m} y^{n} z^{p}: m, n, p=0,1\right\}$, the set of monomials in $x, y, z$ in (3.1), the elements of the matrix, $M^{(7)}$ corresponding to (3.2), can be specified by

$$
\begin{aligned}
M_{\alpha i, \beta}^{(7)} & =h_{i}^{\gamma} \equiv \partial_{\gamma} h_{i}, & & \text { if } \beta=\alpha \gamma, \gamma \in B_{3}, \\
& =0, & & \text { if } \beta \notin \alpha B_{3},
\end{aligned}
$$

where $\partial_{\gamma}=\partial_{x}^{m} \partial_{y}^{n} \partial_{z}^{p}$ for $\gamma=x^{m} y^{n} z^{p}$. Then

$$
\operatorname{deg} M_{\alpha i, \beta}^{(7)}=i+\operatorname{deg} \alpha-\operatorname{deg} \beta,
$$

with $M_{\alpha i, \beta}^{(7)}=0$ if $\operatorname{deg} \beta-\operatorname{deg} \alpha=\operatorname{deg} \gamma>i$. It follows that $\Delta_{7}=\operatorname{det} M^{(7)}$ is homogeneous of degree

$$
\sum_{i=1}^{4} \sum_{\alpha \in C_{2}}(i+\operatorname{deg} \alpha)-\sum_{\beta \in C_{3}} \operatorname{deg} \beta=6 \times 10+4 \times 9-72=24
$$

in $x, y, z, u, v$. Again, since no element of $M^{(7)}$ is more than linear in $v$, the term in $v^{24}$ must come from the product of linear factors. It is straightforward to see that there is only one such product contributing to $\Delta_{7}$, namely, written in order of rows,

$$
h_{1} h_{2}^{z} h_{3}^{y z} h_{4}^{x y z} h_{1} h_{2}^{y} h_{3}^{y z} h_{4}^{x y z} h_{1} h_{2}^{z} h_{3}^{z x} h_{4}^{x y z} h_{1} h_{2}^{y} h_{3}^{x y} h_{4}^{x y z} h_{1} h_{2}^{x} h_{3}^{z x} h_{4}^{x y z} h_{1} h_{2}^{x} h_{3}^{x y} h_{4}^{x y z}
$$

and so the coefficient of $v^{24}$ in $\Delta_{7}$ is

$$
\sigma_{6}^{6} \sigma_{26}^{2} \sigma_{36}^{2} \sigma_{46}^{2} \sigma_{236}^{2} \sigma_{346}^{2} \sigma_{246}^{2} \sigma_{2346}^{6} \text {. }
$$

[For further details, see section 4.3.] In particular, it follows that $M^{(7)}$ is non-singular, or, equivalently, the equations $\alpha h_{m}=0, \alpha \in C_{2}, 1 \leq m \leq 4$, are linearly independent, and that thus $\Delta_{7}=0$ is the desired polynomial of order 24 in $u / v$. 


\subsection{Direct demonstration that $\Delta_{7}$ is the required polynomial of degree 24}

So we can see directly from (3.2) that $\Delta_{7}$ vanishes when $h=0$ and is a homogenous polynomial of degree 24 in $x, y, z, u, v$. As in the $N=5,6$, we can show directly that $\Delta_{7}$ is independent of $x, y, z$. For this purpose, it is notationally convenient to write $\boldsymbol{h}$ for the $4 \times 2$ matrix $\left(h, h^{z}\right)$, and $\boldsymbol{h}^{\gamma}=\partial_{\gamma} \boldsymbol{h}, \gamma=x, y$ or $x y$.

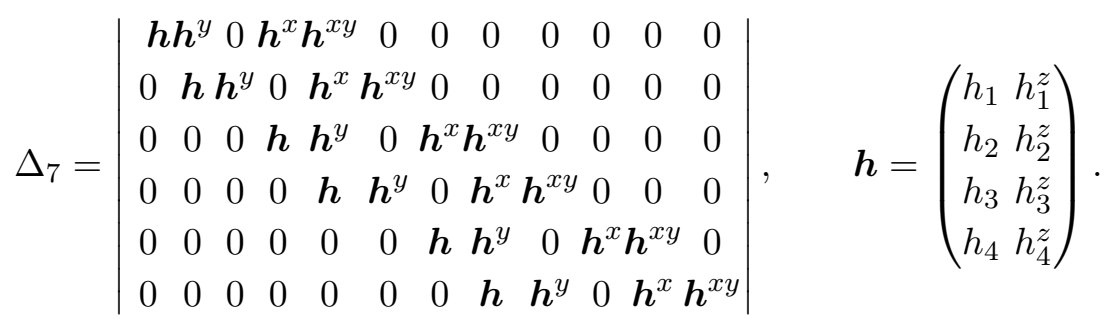

It follows immediately that $\partial_{z} \Delta_{7}=0$. We can show $\partial_{y} \Delta_{7}=\partial_{x} \Delta_{7}=0$ using the same method that we used to show $\partial_{x} \Delta_{6}=0$. To show $\Delta_{7}$ is independent of $y$, first take $y$ times the $n$-th column from the $(n-1)$-th, for $n=3,6,9,12$, and then do the same for $n=2,5,8,11$, leading to

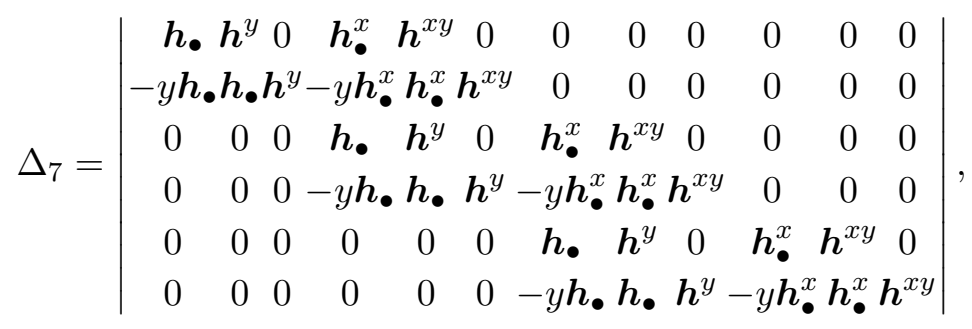

where $\boldsymbol{h}_{\bullet}=\boldsymbol{h}-y \boldsymbol{h}^{y}, \boldsymbol{h}_{\bullet}^{x}=\boldsymbol{h}^{x}-y \boldsymbol{h}^{x y}$, which are independent of $y$, by linearity. Now add $y$ times the $n$-th row to the $(n+1)$-th row for $n=1,3,5$, and then again subtract the $n$-th column from the $(n-1)$-th, for $n=3,6,9,12$, leading to

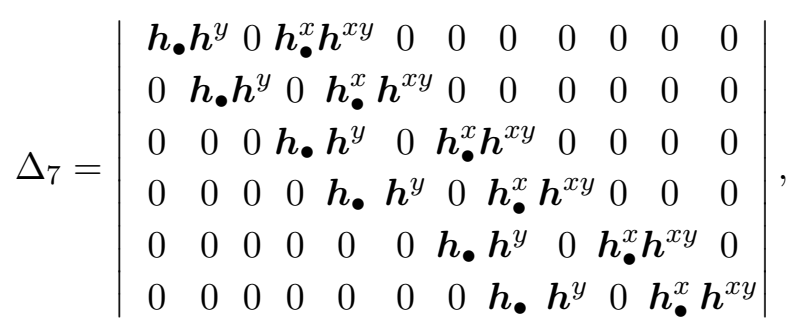

which is manifestly independent of $y$. A similar, but longer, argument shows that $\partial_{x} \Delta_{7}=$ 0 . Thus we have shown directly that $\Delta_{7}$, as defined by (3.2), (i) vanishes when $h_{1}=$ $h_{2}=h_{3}=h_{4}=0$; (ii) is a homogeneous 24th order polynomial; and (iii) is independent of $x, y, z$, and so provides the required polynomial obtained by eliminating $x, y, z$, from $h_{1}=h_{2}=h_{3}=h_{4}=0$. 


\section{Elimination theory for general $N$}

\subsection{Construction of the $(N-3)$ !-th degree polynomial $\Delta_{N}$ using elimination theory}

For $N \geq 5$, write $x_{a}=z_{a+1}, 1 \leq a \leq N-4, u=z_{N-2}, v=z_{N-1}$. We seek to eliminate $x_{a}, 2 \leq a \leq N-3$, between the equations $h_{m}=0,1 \leq m \leq N-3$. Following the approach adopted in the $N=6,7$ cases, we consider the $(N-3)$ ! linear relations

$$
h_{m} \prod_{a=1}^{N-5} x_{a}^{m_{a}}=0, \quad 1 \leq m \leq N-3, \quad 0 \leq m_{a} \leq N-4-a, \quad 1 \leq a \leq N-5,
$$

between the variables

$$
\prod_{a=1}^{N-4} x_{a}^{m_{a}}=0, \quad 0 \leq m_{a} \leq N-3-a, \quad 1 \leq a \leq N-4,
$$

that is $(N-3) !-1$ variables other than 1 . So, provided that the relations (4.1) are independent, we can use them to eliminate the $x_{a}, 2 \leq a \leq N-3$, and obtain a polynomial equation for $u / v$.

Let

$$
\begin{aligned}
& C_{M}=\left\{\prod_{a=1}^{M} x_{a}^{m_{a}}: 0 \leq m_{a} \leq M-a+1,1 \leq a \leq M\right\}, \\
& R_{M}=\left\{(\alpha, m): \alpha \in C_{M-1}, 1 \leq m \leq M+1\right\},
\end{aligned}
$$

and

$$
B_{M}=\left\{\prod_{a=1}^{M} x_{a}^{m_{a}}: 0 \leq m_{a} \leq 1,1 \leq a \leq M\right\} .
$$

Note $\left|C_{M}\right|=(M+1) !,\left|B_{M}\right|=2^{M}$, and

$$
d_{M}=\sum_{\alpha \in C_{M}} \operatorname{deg} \alpha=\frac{M(M+1)}{4}(M+1) !
$$

which follows because $d_{M}$ satisfies the recurrence relation

$$
d_{M+1}=(M+2) d_{M}+\frac{1}{2}(M+1)(M+2)\left|C_{M}\right| .
$$

The relations (4.1) are labelled by $(\alpha, m) \in R_{N-4}$,

$$
\alpha h_{m}=\sum_{\gamma \in B_{N-4}} \alpha \gamma h_{m}^{\gamma}=\sum_{\beta \in C_{N-4}} M_{\alpha m, \beta}^{(N)} \beta, \quad \alpha \in C_{N-5}, 1 \leq m \leq N-3,
$$

where, for $\alpha \in C_{N-5}, \beta \in C_{N-4}$,

$$
\begin{aligned}
M_{\alpha m, \beta}^{(N)} & =h_{m}^{\gamma} & & \text { if } \beta=\alpha \gamma, \gamma \in B_{N-4}, \\
& =0 & & \text { if } \beta \notin \alpha B_{N-4},
\end{aligned}
$$


with

$$
\operatorname{deg} M_{\alpha m, \beta}^{(N)}=m+\operatorname{deg} \alpha-\operatorname{deg} \beta,
$$

and $M_{\alpha m, \beta}^{(N)}=0$ if $\operatorname{deg} \beta-\operatorname{deg} \alpha=\operatorname{deg} \gamma>m$.

The consistency condition is given by the vanishing of

$$
\Delta_{N}=\operatorname{det} M^{(N)} .
$$

Here, in principle, the $h_{m}^{\gamma}$ are evaluated at $x_{a}=0,1 \leq a \leq N-4$, but, by the argument used for $N=5,6,7$, the condition $\Delta_{N}=0$ is independent of the $x_{a}$.

From (4.10) it follows that $\Delta_{N}$ is a homogeneous polynomial of degree $\delta_{N}$, where

$$
\begin{aligned}
\delta_{N} & =\sum_{m=1}^{N-3} \sum_{\alpha \in C_{N-5}}(m+\operatorname{deg} \alpha)-\sum_{\beta \in C_{N-4}} \operatorname{deg} \beta \\
& =\frac{(N-3)(N-2)}{2}\left|C_{N-5}\right|+(N-3) d_{N-5}-d_{N-4}
\end{aligned}
$$

from which it follows that $\delta_{N}=(N-3)$ !, the size of $M^{(N)}$. As for $N=6,7$, since the elements $M_{\alpha m, \beta}^{(N)}$ are at most linear in $v$, and a contribution to the coefficient of $v^{\delta_{N}}$ can only come from a product of elements of degree one, $M_{\alpha m, \beta}^{(N)}=h_{m}^{\gamma}$, with $\operatorname{deg} \gamma=\operatorname{deg} \beta-\operatorname{deg} \alpha=$ $m-1$.

\subsection{Direct demonstration that $\Delta_{N}$ is the required polynomial}

Again, write $h=\left(h_{1}, h_{2}, \ldots, h_{N-3}\right)^{T}$. First, we can show directly that $\Delta_{N}=0$ vanishes when $h=0$ because, from (4.9), in the first column of $M^{(N)}$,

$$
M_{\alpha m, 1}^{(N)}=h_{m}, \quad \text { if } \alpha=1 ; \quad M_{\alpha m, 1}^{(N)}=0, \quad \text { if } \alpha \neq 1,
$$

and so the whole first column of $M^{(N)}$ vanishes when $h=0$, implying that $\Delta_{N}=$ $\operatorname{det} M^{(N)}=0$ when $h=0$.

We have already established that it is a homogeneous polynomial of degree $\delta_{N}$ and we now wish to show that it is independent of $x_{b}, 1 \leq b \leq N-4$. We do this by generalizing the argument of section 3.2. Let

$$
C_{M}^{b}=\left\{\prod_{\substack{a=1 \\ a \neq b}}^{M} x_{a}^{m_{a}}: 0 \leq m_{a} \leq M-a+1,1 \leq a \leq M, a \neq b\right\}
$$

and

$$
B_{M}^{b}=\left\{\prod_{\substack{a=1 \\ a \neq b}}^{M} x_{a}^{m_{a}}: 0 \leq m_{a} \leq 1,1 \leq a \leq M, a \neq b\right\} .
$$

for $1 \leq b \leq M$. Then, the non-zero elements of the column of $M^{(N)}$ specified by

$$
\beta=x_{b}^{m_{b}} \tilde{\beta} \in C_{N-4}, \quad \tilde{\beta} \in C_{N-4}^{b}, \quad 0 \leq m_{b} \leq N-b-3 \equiv m_{b}^{*},
$$


are in the rows specified by $(\alpha, m)$, where: $1 \leq m \leq N-3 ; \alpha=x_{b}^{m_{b}^{\prime}} \tilde{\alpha}$, and $m_{b}^{\prime}=0$ if $m_{b}=0, m_{b}^{\prime}=m_{b}$ or $m_{b}^{\prime}=m_{b}-1$ if $0<m_{b}<m_{b}^{*}$, and $m_{b}^{\prime}=m_{b}^{*}-1$ if $m_{b}=m_{b}^{*}$; and $\tilde{\beta}=\tilde{\alpha} \tilde{\gamma}$ for some $\tilde{\gamma} \in B_{N-4}$. Then

$$
M_{\alpha m, \beta}^{(N)}=h_{m}^{\gamma}, \quad \gamma=x_{b}^{m_{b}-m_{b}^{\prime}} \tilde{\gamma}
$$

For given $\tilde{\beta} \in C_{N-4}^{b}$, we replace successively subtract $x_{b}$ times the column of $M^{(N)}$ labelled by $\beta=x_{b}^{m_{b}^{*}-r} \tilde{\beta}$ from that labelled by $\beta=x_{b}^{m_{b}^{*}-r-1} \tilde{\beta}$ for $r=0,1, \ldots, m_{b}^{*}-1$. By this process, the matrix $M^{(N)}$ is transformed into a matrix $M^{(N, 1)}$ (We suppress the dependence on $b$.) with the non-zero elements in the column labelled by $\beta$ occurring in the rows labelled by $(\alpha, m)$ where: $1 \leq m \leq N-3 ; \alpha=x_{b}^{m_{b}^{\prime}} \tilde{\alpha}$, with $\max \left(0, m_{b}-1\right) \leq m_{b}^{\prime} \leq m_{b}^{*}-1$. Then

$$
\begin{aligned}
M_{\alpha m, \beta}^{(N, 1)} & =h_{m}^{x_{b} \tilde{\gamma}} & & \text { if } m_{b}^{\prime}=m_{b}-1, \\
& =\left(-x_{b}\right)^{m_{b}^{\prime}-m_{b}} h_{m \bullet}^{\tilde{\gamma}}, & & \text { if } m_{b}-1<m_{b}^{\prime} \leq m_{b}^{*}-1,
\end{aligned}
$$

where $h_{m}^{\tilde{\gamma}}=h_{m}^{\tilde{\gamma}}-x_{b} h_{m}^{x_{b} \tilde{\gamma}}$, so that $\partial_{x_{b}} h_{m}^{\tilde{\gamma}} \bullet=0$. It follows from the construction that $\operatorname{det} M^{(N, 1)}=\operatorname{det} M^{(N)}=\Delta_{N}$.

Next add $x_{b}$ times the row labelled by $\left(x_{b}^{m_{b}^{*}-r-2} \tilde{\alpha}, m\right)$ to that labelled by $\left(x_{b}^{m_{b}^{*}-r-1} \tilde{\alpha}, m\right)$ for $r=0,1, \ldots, m_{b}^{*}-2$. By this process, the matrix $M^{(N, 1)}$ is transformed into a matrix $M^{(N, 2)}$ with

$$
M_{\alpha m, \beta}^{(N, 2)}=M_{\alpha m, \beta}^{(N)}, \quad \text { if } m_{b}>0 ; \quad M_{\alpha m, \beta}^{(N, 2)}=M_{\alpha m, \beta}^{(N)}-x_{b} \partial_{x_{b}} M_{\alpha m, \beta}^{(N)} \quad \text { if } m_{b}=0,
$$

and $\operatorname{det} M^{(N, 2)}=\operatorname{det} M^{(N, 1)}=\Delta_{N}$. Now applying these two processes successively to $M^{(N, 2)}$, but stopping at $r=m_{b}^{*}-2$ in the first process and $r=m_{b}^{*}-3$ in the second, we obtain another matrix $M^{(N, 4)}$, such that

$$
M_{\alpha m, \beta}^{(N, 4)}=M_{\alpha m, \beta}^{(N)}, \quad \text { if } m_{b}>1 ; \quad M_{\alpha m, \beta}^{(N, 4)}=M_{\alpha m, \beta}^{(N)}-x_{b} \partial_{x_{b}} M_{\alpha m, \beta}^{(N)} \quad \text { if } m_{b}=0,1,
$$

$\operatorname{det} M^{(N, 4)}=\Delta_{N}$. After performing $m_{b}^{*}$ cycles of this process, we obtain a matrix $M^{\left(N, 2 m_{b}^{*}\right)}$ all of whose elements are independent of $x_{b}$ and whose determinant equals $\Delta_{N}$, showing that this also is independent of $x_{b}$. It follows that $\Delta_{N}$ has the desired properties of being a homogeneous polynomial of degree $\delta_{N}$ in $u, v$, independent of $x_{b}, 1 \leq b \leq N-4$, vanishing when $h=0$.

To complete the proof that this polynomial does indeed determine the solutions, we need to show that it does not vanish identically. To do this, we show in section 4.3 that only one product in the expression for the determinant $\Delta_{N}$ contributes to the coefficient of the leading power, $v^{\delta_{N}}$.

\subsection{The leading power of $v, v^{\delta_{N}}$}

So far we have established that $\Delta_{N}=\operatorname{det} M^{(N)}$ is homogeneous of degree $\delta_{N}=(N-3)$ ! in $u, v$, and is independent of $x_{a}, 1 \leq a \leq N-4$. Thus, to evaluate $\Delta_{N}$, we can choose eventually to put $x_{a}=0,1 \leq a \leq N-4$. Because $h_{m}^{\gamma}$ vanishes when $x_{a}=0,1 \leq a \leq N-4$, if $\operatorname{deg} h_{m}^{\gamma}>2$, the only contributions to $\operatorname{det} M^{(N)}$ come from elements $M_{\alpha m, \beta}^{(N)}=h_{m}^{\gamma}$ with 
$\operatorname{deg} h_{m}^{\gamma}=m-\operatorname{deg} \gamma=0,1,2$, which are constant, linear in $u, v$, and proportional to $u v$, respectively. Since each product of $\delta_{N}$ elements of $M^{(N)}$, contributing to the determinant is homogeneous in $u, v$ of degree $\delta_{N}$, the only products that contribute to the leading term proportional to $v^{\delta_{N}}$ are ones in which all the factors are of degree 1 . These are of the form $h_{m}^{\gamma}=\partial_{\gamma} h_{m}$, where $\operatorname{deg} \gamma=m-1$. There are $\left(\begin{array}{c}N-4 \\ m-1\end{array}\right)$ such elements $M_{\alpha m, \beta}^{(N)}$ in the row $(\alpha, m) \in R_{N-4}$, occuring in the columns $\beta=\alpha \gamma$, where $\operatorname{deg} \gamma=m-1$.

To calculate the coefficient of $v^{\delta_{N}}$, let $\widetilde{M}_{\alpha m, \beta}^{(N)}=M_{\alpha m, \beta}^{(N)}$ if $\operatorname{deg} M_{\alpha m, \beta}^{(N)}=1$, and zero otherwise; then the coefficient of $v^{\delta_{N}}$ in $\operatorname{det} M^{(N)}$ is the same as in $\operatorname{det} \widetilde{M}^{(N)}$. Now, $\widetilde{M}_{\alpha m, \beta}^{(N)}=$ 0 is zero unless $\operatorname{deg} \beta=\operatorname{deg} \alpha+m-1$, and, if we divide the rows and the columns into subsets

$$
R_{N-4}^{j}=\left\{(\alpha, m) \in R_{N-4}: \operatorname{deg} \alpha+m-1=j\right\}, \quad C_{N-4}^{j}=\left\{\beta \in C_{N-4}: \operatorname{deg} \beta=j\right\},
$$

$0 \leq j \leq d_{N}=\frac{1}{2}(N-3)(N-4)$, the nonzero elements of $\widetilde{M}^{(N)}$ fall into $d_{N}+1$ blocks, $\widetilde{M}_{N}^{j}$, with rows labelled by $(\alpha, m) \in R_{N-4}^{j}$ and columns by $\beta \in C_{N-4}^{j}, 0 \leq j \leq d_{N}$. These blocks are square with dimension $d_{N j}$, where

$$
\prod_{a=1}^{N-4}\left(1+x+\ldots+x^{a}\right)=\sum_{j=0}^{d_{N}} d_{N j} x^{j}=(1-x)^{4-N} \prod_{a=1}^{N-4}\left(1-x^{a+1}\right) .
$$

Note that putting $x=1$ in this relation verifies that

$$
\sum_{j=0}^{d_{N}} d_{N j}=(N-3) !=\left|R_{N-4}\right|=\left|C_{N-4}\right| .
$$

A non-zero contribution to $\operatorname{det} M^{(N)}$ corresponds to a map $\phi: R_{N-4} \rightarrow C_{N-4}$, which is bijective, such that

$$
\phi(\alpha, m)=\gamma \alpha, \quad \text { with } \quad \gamma \in B_{N-4} \quad \text { and } \quad \operatorname{deg} \gamma=m-1 .
$$

We will show that there is precisely one such map.

If $m=1$, then $\operatorname{deg} \gamma=0$, so that $\gamma=1$, and hence $\phi(\alpha, 1)=\alpha$, for all $\alpha \in C_{N-5}$.

It follows from the block structure of $\widetilde{M}^{(N)}$ that any such $\phi$ defines bijective maps $R_{N-4}^{j} \rightarrow C_{N-4}^{j}$, and so our task is equivalent to showing that, for each $0 \leq j \leq d_{N}$, there is one exactly one bijective map $\phi: R_{N-4}^{j} \rightarrow C_{N-4}^{j}$ with the property [*]. In this case, we shall say the block $\widetilde{M}_{N}^{j}$ has the property [*].

We use induction to establish that $\widetilde{M}_{N}^{j}$ has the property [*]. To this end, we divide $C_{N-4}^{j}$ and $R_{N-4}^{j}$ into subsets $C_{N-4}^{j, k}$ and $R_{N-4}^{j, k}$, respectively. We define

$$
C_{n}^{j, k}=\left\{\beta \in C_{n}^{j}: x_{n-k+1}^{k} \mid \beta ; x_{a}^{n-a+1} \not \beta, 1 \leq a \leq n-k\right\}
$$

so that $C_{n}^{j, k}$ comprises elements $\beta$, which are the product of factors $x_{n-a+1}^{m_{a}}, 1 \leq a \leq n$, where $0 \leq m_{a}<a$ if $1 \leq a \leq n-k, m_{n-k+1}=k$, and $0 \leq m_{a} \leq a$ if $n-k+2 \leq a \leq n$. It follows that

$$
C_{n}^{j, k}=\left\{x_{n-k+1}^{k} \beta^{\prime}: \beta^{\prime} \in C_{n-1}^{j-k}\left(x_{1}, \ldots, x_{n-k}, x_{n-k+2}, \ldots, x_{n}\right)\right\} \equiv x_{n-k+1}^{k} C_{n-1}^{j-k}
$$


For given $N, C_{N-4}^{j}$ is the disjoint union of $C_{N-4}^{j}, 0 \leq j \leq d_{N}=\frac{1}{2}(N-3)(N-4)$; for given $N, j, C_{N-4}^{j}$ is the disjoint union of $C_{N-4}^{j, k}$, where the range of $k$ is limited above by both $j$ and $N-4$, and below by 0 and the requirement that $j-k \leq d_{N-1}$ in order that $C_{N-4}^{j, k}=x_{N-k-3}^{k} C_{N-5}^{j-k}$ be nonempty, so the range is $\max \left(0, j-d_{N-1}\right) \leq k \leq \min (j, N-4)$. Note that, in particular, $C_{N-4}^{j, 0}=C_{N-5}^{j}$.

Write

$$
\begin{aligned}
& R_{n}^{j, 0}=\left\{(\alpha, 1): \alpha \in C_{n-1}, \operatorname{deg} \alpha=j\right\} \subset R_{n}^{j}, \\
& R_{n}^{j, k}=\left\{(\alpha, m) \in R_{n}^{j}: 1<m \leq n+1 ; x_{n-k+1}^{k-1} \mid \alpha ; x_{a}^{n-a} \not \alpha, 1 \leq a \leq n-k\right\}, \quad 1 \leq k \leq j .
\end{aligned}
$$

Then $R_{n}^{j, k}=\varnothing$ if $k<j-d_{N-1}$ or $k>N-4$; and $R_{n}^{j}$ is the union of $R_{n}^{j, k}$ for $\max \left(0, j-d_{N-1}\right) \leq k \leq \min (j, N-4)$. Note $\left|R_{n}^{j, k}\right|=\left|C_{n}^{j, k}\right|$ and

$$
\begin{array}{r}
R_{n}^{j, k}=\left\{\left(x_{n-k+1}^{k-1} \alpha^{\prime}, m^{\prime}+1\right):\left(\alpha^{\prime}, m^{\prime}\right) \in R_{n-1}^{j-k}\left(x_{1}, \ldots, x_{n-k}, x_{n-k+2}, \ldots, x_{n-1}\right)\right\} \\
1 \leq k \leq j .
\end{array}
$$

If $(\alpha, m) \in R_{n}^{j, k}$, with $k>0$, then $x_{a}^{n-a} \gamma \alpha, 1 \leq a \leq n-k$. Suppose $\phi(\alpha, m)=\gamma \alpha$, where $\gamma \in B_{n}$. Then $x_{a}^{n-a+1} \vee \gamma \alpha, 1 \leq a \leq n-k$, for $\gamma$ can add at most one factor of $x_{a}$ to $\alpha$. Thus $\phi(\alpha, m) \notin C_{n}^{j, n-a+1}, 1 \leq a \leq n-k$, i.e. $\phi(\alpha, m) \notin C_{n}^{j, \ell}, k+1 \leq \ell \leq n$. Since $\phi\left(R_{n}^{j}\right)=C_{n}^{j}$ and $\phi\left(R_{n}^{j, 0}\right)=C_{n}^{j, 0}$, because we always have $\phi(\alpha, 1)=\alpha$, it follows that

$$
\phi\left(R_{n}^{j, k}\right) \subset \bigcup_{\ell=1}^{k} C_{n}^{j, \ell} .
$$

In particular $\phi\left(R_{n}^{j, 1}\right) \subset C_{n}^{j, 1}$, and since $\left|R_{n}^{j, 1}\right|=\left|C_{n}^{j, 1}\right|$ and $\phi$ is bijective, it follows that $\phi\left(R_{n}^{j, 1}\right)=C_{n}^{j, 1}$, and similarly, by induction, that $\phi\left(R_{n}^{j, k}\right)=C_{n}^{j, k}$, for all $k$.

Given

$$
\phi^{\prime}: R_{n-1}^{j-k}\left(x_{1}, \ldots, x_{n-k}, x_{n-k+2}, \ldots, x_{n-1}\right) \rightarrow C_{n-1}^{j-k}\left(x_{1}, \ldots, x_{n-k}, x_{n-k+2}, \ldots, x_{n}\right),
$$

with the property $[*]$, setting $\phi\left(x_{n-k+1}^{k-1} \alpha^{\prime}, m^{\prime}+1\right)=x_{n-k+1}^{k} \phi^{\prime}\left(\alpha^{\prime}, m^{\prime}\right)$ defines a map

$$
\phi: R_{n}^{j, k}\left(x_{1}, \ldots, x_{n-1}\right) \rightarrow C_{n}^{j, k}\left(x_{1}, \ldots, x_{n}\right),
$$

such that, if $\phi^{\prime}\left(\alpha^{\prime}, m^{\prime}\right)=\gamma^{\prime} \alpha^{\prime}, \gamma^{\prime} \in B_{n-1}\left(x_{1}, \ldots, x_{n-1}\right)$, then $\phi(\alpha, m)=\gamma \alpha$, with $\gamma=$ $x_{n-k+1} \gamma^{\prime}$. So $\phi$ has the property [*] if $\phi^{\prime}$ does. Conversely, given $\phi: R_{n}^{j, k} \rightarrow C_{n}^{j, k}$ with the property [*], we can construct $\phi^{\prime}: R_{n-1}^{j-k} \rightarrow C_{n-1}^{j-k}$ with the property [*], and so the uniqueness of a map $\phi^{\prime}: R_{n-1}^{j-k} \rightarrow C_{n-1}^{j-k}$ with the property [*] implies that for a map $\phi: R_{n}^{j, k} \rightarrow C_{n}^{j, k}$ with the property (and conversely). The desired result follows by induction.

Dividing $C_{N-4}^{j}$ and $R_{N-4}^{j}$ into subsets $C_{N-4}^{j, k} \cong x_{N-k-3}^{k} C_{N-5}^{j-k}$ and $R_{N-4}^{j, k} \cong$ $x_{N-k-3}^{k} R_{N-5}^{j-k}$, respectively, and subdividing further and so on inductively defines orders on $C_{N-4}^{j}$ and $R_{N-4}^{j}$, with respect to which $\phi$ is lower triangular, and, consequently, reordering the rows and columns of $\widetilde{M}^{(N)}$ brings it into a form which is lower triangular. 
It follows that $\operatorname{det} \widetilde{M}^{(N)}$, which contains the term in $\Delta_{N}$ involving $v^{\delta_{N}}$, is given, up to sign, by a single product of the $(N-3)$ ! diagonal elements (after reordering as above), which are of the form $h_{m}^{\gamma}$, where $\gamma \in B_{N-4}$ and $m=\operatorname{deg} \gamma+1$, with each $m$ occurring $(N-4)$ ! times. It follows immediately from this that the coefficient of $v^{\delta_{N}}$ is nonzero provided that all the Mandelstam invariants $\sigma_{S} \neq 0$.

To evaluate this coefficient more explicitly, consider further $\operatorname{det} \widetilde{M}^{(N)}$. Up to sign, it is given by a product of the form

$$
\prod_{\gamma \in B_{N-4}}\left[h_{d_{\gamma}}^{\gamma}\right]^{n_{\gamma}}, \quad \text { where } \quad d_{\gamma}=\operatorname{deg} \gamma+1
$$

and the sum of the $n_{\gamma}$ for a given value of $\operatorname{deg} \gamma$ is $(N-4)$ !. The number of $\gamma \in B_{N-4}$ with a given value of $\operatorname{deg} \gamma$ is $(N-4) ! /(N-4-\operatorname{deg} \gamma) !(\operatorname{deg} \gamma)$ ! and so, if the result is unchanged under permutation of the variables $x_{a}, 1 \leq a \leq N-4$, with respect to which derivatives are taken, $n_{\gamma}$ will only depend on $\operatorname{deg} \gamma$ and we shall have

$$
n_{\gamma}=(N-4-\operatorname{deg} \gamma) !(\operatorname{deg} \gamma) !
$$

From this it would follow that the coefficient of $v^{\delta_{N}}$ is

$$
\prod_{r=0}^{N-4} \prod_{\substack{S \subset A^{\prime \prime} \\|S|=r}} \sigma_{S_{M}}^{n_{r}}, \quad \text { where } \quad S_{M}=S \cup\{N-1\}, \quad n_{r}=r !(N-4-r) \text { ! }
$$

where $A^{\prime \prime}=\{a: 1<a<N-2\}$.

We can establish (4.34) directly from our iterative construction of the map $\phi$. From this construction, if, inductively, $\operatorname{det} \widetilde{M}^{(N)}$ is given by (4.33), up to a sign, $\operatorname{det} \widetilde{M}^{(N+1)}$ is given by

$$
\left[h_{1}\right]^{(N-3) !} \prod_{i=1}^{N-3} \prod_{\gamma \in B_{N-4}^{i}}\left[h_{d_{\gamma}+1}^{x_{i} \gamma}\right]^{n_{\gamma}}
$$

where

$$
B_{N-4}^{i}=\left\{\prod_{a=1, a \neq i}^{N-3} x_{a}^{m_{a}}: 0 \leq m_{a} \leq 1,1 \leq a \leq N-3\right\} .
$$

Then (4.36) is exactly what we get from (4.33) and (4.34), replacing $N-4$ by $N-3$, so establishing the result by induction.

\section{Resultants, hyperdeterminants and Hilbert series}

\subsection{Sparse resultants}

It is the special feature of the polynomial scattering equations,

$$
h_{m}=0, \quad 1 \leq m \leq N-3,
$$


that the $h_{m}$ are linear and homogeneous in the variables $z_{a}, 2 \leq a \leq N-1$, and it is this linearity that ensures that we can obtain relatively simple explicit forms for the equation $\Delta_{N}=0$, obtained by eliminating

$$
x_{a}=z_{a+1}, \quad 1 \leq a \leq N-4,
$$

in favor of $u=z_{N-2}, v=z_{N-1}$, while it is the fact that $\operatorname{deg} h_{m}=m, 1 \leq m \leq N-3$, that implies that the $\operatorname{deg} \Delta_{N}=\delta_{N}=(N-3)$ ! viewed as a polynomial in $u / v$.

$\Delta_{N}$ is the resultant of the $N-3$ equations (5.1), viewed as polynomial equations in the $N-4$ variables (5.2), that is the polynomial in the coefficients of these polynomials (which are combinations of factors $\sigma_{S}, u$ and $v$ ) whose vanishing is the necessary and sufficient condition for these equations (5.1) to have a common solution for the variables (5.2), taking values on the Riemann sphere. The theory of resultants, in the two variable case, has its origins in the work of Sylvester [28] and Cayley [29], extended to the multivariable case by Macauley [36], and revived and set in a modern context by Jouanolou [37] and Gel'fand, Kapranov and Zelevinsky (GKZ) [31]. For accounts, see, e.g., chapter 4 of [32] or chapter 3 of [38].

The existence of the resultant, of a set of $M+1$ polynomial equations in $M$ variables, as an irreducible polynomial in the coefficients of the equations with integral coefficients, unique up to sign, can be established using techniques of algebraic geometry (see [31] and $[37,38])$. However, these results do not provide explicit expressions for the resultant when $M>1$. Moreover, these existence theorems are typically formulated for general polynomials of a particular degree, involving all its coefficients, and they can vanish identically when one specializes to polynomials in which certain terms are absent. This is the situation for the scattering equations (5.1), where the condition of linearity in each of the variables (5.2) means that many terms are absent from the general form of a multinomial of the same degree.

Although the theory of resultants for general polynomials can not be directly applied to the scattering equations, the theory of sparse resultants, developed to deal with situations in which some of the monomial terms in the polynomial equations considered are absent, precisely addresses their case (see, e.g., section 4.3 of [32]) While a complete theory of sparse resultants is lacking, the scattering equations fall into a category for which some precise and explicit results exist [31, 39-41]. In particular, it follows from the discussion of chapter 13 in [31] that the (sparse) resultant of the multilinear equations (5.1), viewed as polynomials in the variables (5.2), defined as above, exists and, by Proposition 2.1 of that chapter, has degree is $(N-3)$ ! (see also [40]). Because $\Delta_{N}$, constructed as in section 4.1, vanishes when (5.1) holds, has this degree and has no integer factors, it must indeed be this sparse resultant (which is unique up to sign), and so irreducible as a polynomial. Consequently, there is a solution to (5.1) for (5.2) for each of the $\delta_{N}$ roots of $\Delta_{N}=0$ for $u / v$.

\subsection{Hyperdeterminants and the scattering equations}

Gel'fand, Kapranov and Zelevinsky [31] relate their discussion of sparse resultants to a theory of multidimensional or hyperdeterminants, which they have developed in this 
context. This theory generalizes the familiar concept of the determinant of a (twodimensional) square matrix, $\mathcal{M}_{i_{0} i_{1}}, 0 \leq i_{0}, i_{1} \leq \ell$, of shape $(\ell+1) \times(\ell+1)$, to an $(M+1)$ dimensional matrix or array, $\mathcal{M}_{i_{0} i_{1} \cdots i_{M}}$, where $0 \leq i_{j} \leq \ell_{j}, 0 \leq j \leq M$, of shape or format $\left(\ell_{0}+1\right) \times\left(\ell_{1}+1\right) \times \cdots \times\left(\ell_{M}+1\right)$, and we take $\ell_{0}$ to be the largest of the $\ell_{j}$, that is $\ell_{j} \leq \ell_{0}, 1 \leq j \leq M$. Associated to this multidimensional matrix are the multilinear functions

$$
f_{i_{0}}\left(\xi_{1}, \ldots, \xi_{M}\right)=\mathcal{M}_{i_{0} i_{1} \cdots i_{M}} \xi_{1}^{i_{1}} \ldots \xi_{M}^{i_{M}}, \quad 0 \leq i_{0} \leq \ell_{0},
$$

where implicitly $i_{j}$ is summed over $0 \leq i_{j} \leq \ell_{j}$, and $\xi_{j} \in \mathbb{C}^{\ell_{j}}, 1 \leq j \leq M$.

The hyperdeterminant, $\operatorname{det} \mathcal{M}$, is defined as the (sparse) resultant of the $\ell_{0}+1$ homogeneous equations

$$
f_{i_{0}}\left(\xi_{1}, \ldots, \xi_{M}\right)=0, \quad 0 \leq i_{0} \leq \ell_{0},
$$

that is the polynomial in the coefficients of the $f_{i_{0}}$ (the elements of the multidimensional matrix $\mathcal{M}$ ) whose vanishing is the necessary and sufficient condition for (5.4) to have a nontrivial solution for the $\xi_{j}$, which we should regard as elements of $\mathbb{C P}^{\ell_{j}}$, because of the multihomogeneity of (5.3). $\operatorname{det} \mathcal{M}$ itself is nontrivial if and only if $\ell_{0} \leq \ell_{1}+\cdots+\ell_{M}$, for otherwise the $\xi_{j} \in \mathbb{C P}^{\ell_{j}}$ are always overdetermined. This definition of $\operatorname{det} \mathcal{M}$ agrees with the conventional definition in the two-dimensional case.

Explicit formulae for $\operatorname{det} \mathcal{M}$ in the general case are not known, but an exception is the borderline case, when $\ell_{0}=\ell_{1}+\cdots+\ell_{M}$; in this case $\mathcal{M}$ is said to be of boundary format, there is a simple expression for the degree, $\operatorname{deg} \mathcal{M}=\left(\ell_{0}+1\right) ! / \ell_{1} ! \cdots \ell_{M} !$, and $\operatorname{det} \mathcal{M}$ can constructed as a (two-dimensional) determinant, using methods similar to those of sections 3 and 4 .

$\Delta_{N}$ is the hyperdeterminant of a multidimensional matrix of boundary format, $(N-$ $3) \times 2 \times \cdots \times 2$, where there are $M=N-4$ factors of 2 , i.e. $\ell_{0}=N-4, \ell_{1}=1, \ldots \ell_{N-4}=1$. To show this, we homogenize $h_{m}$ as a function of the variables (5.2) by setting $x_{a}=\xi_{a}^{1} / \xi_{a}^{0}$, and defining

$$
f_{m-1}\left(\xi_{1}, \ldots, \xi_{M}\right)=h_{m}\left(\xi_{1}^{1} / \xi_{1}^{0}, \ldots, \xi_{M}^{1} / \xi_{M}^{0}, u, v\right) \prod_{a=1}^{M} \xi_{a}^{0}, \quad 1 \leq m \leq M+1=N-3
$$

so that the $f_{m}$ are linear in each $\xi_{a}$. Then, writing $A^{\vee}=\{a: 1 \leq a \leq N-4\}$,

$$
\begin{aligned}
f_{m-1}\left(\xi_{1}, \ldots, \xi_{M}\right)= & \sum_{\substack{U \subset A \vee \\
|U|=m}} \sigma_{\widetilde{U}} \xi_{U}^{1} \xi_{\bar{U}}^{0}+\sum_{\substack{U \subset A \vee \\
|U|=m-1}} \sigma_{\widetilde{U}_{u}} \xi_{U}^{1} \xi_{\bar{U}}^{0} u \\
& +\sum_{\substack{U \subset A \vee \\
|U|=m-1}} \sigma_{\widetilde{U}_{v}} \xi_{U}^{1} \xi_{\bar{U}}^{0} v+\sum_{\substack{U \subset A^{\vee} \\
|U|=m-2}} \sigma_{\widetilde{U}_{u v}} \xi_{U}^{1} \xi_{\bar{U}}^{0} u v
\end{aligned}
$$

where $\bar{U}$ denotes the complement of $U$ in $A^{\vee}, \widetilde{U}=\{a+1: a \in U\}, \widetilde{U}_{u}=\widetilde{U} \cup\{N-2\}, \widetilde{U}_{v}=$ $\widetilde{U} \cup\{N-1\}$, and $\widetilde{U}_{u v}=\widetilde{U} \cup\{N-2, N-1\}$. This determines a multidimensional matrix $\mathcal{M}$ as in (5.3) with $\ell_{0}=M$ and $\ell_{a}=2,1 \leq a \leq M$. Since the condition that the equations $h_{m}=0$ have solutions for some $x_{a}$ clearly the same as that the equations $f_{m-1}=0$ have solutions for some $\xi_{a}$, it follows that $\Delta_{N}=\operatorname{det} \mathcal{M}$, up to sign. 
We note that the hyperdeterminant corresponding to $N=6$ case is discussed by GKZ as example 4.9 of [39]; and, as Cardona and Kalousios [35] have pointed out, Sturmfels discussed the equations of the $N=7$ case as an example of sparse resultants in section 4.5 of [32].

\subsection{Hilbert series and regular sequences}

The CHY expressions for tree amplitudes in massless field theories are given as residue functions evaluated at the solutions of the scattering equations and summed over those solutions. Thus such a residue function, $\Phi$, only need be specified up the addition of sums of polynomial multiples of the $h_{m}$, because the additional terms vanish at the solutions of the scattering equations.

If $\mathcal{R}$ denotes the ring of polynomials in $z_{a}, 2 \leq a \leq N-1$, and $\mathcal{I} \equiv\left\langle h_{1}, \ldots, h_{N-3}\right\rangle$ denotes the ideal in $\mathcal{R}$ generated by the $h_{m}, 1 \leq m \leq N-3$, that is the polynomials of the form $g_{1} h_{1}+\ldots+g_{N-3} h_{N-3}$, we are interested in $\mathcal{S}=\mathcal{R} / \mathcal{I}$, polynomials modulo elements of $\mathcal{I}$. Further, we consider homogeneous polynomials in the $z_{a}$ of a specific degree, $n$, say. Suppose $\mathcal{R}_{n}$ denotes the homogeneous polynomials of degree $n$, so that $\mathcal{R}$ is the direct sum of the $\mathcal{R}_{n}$; and $\mathcal{I}_{n}$ denotes the elements of $\mathcal{I}$ which are homogeneous of degree $n$, so that $\mathcal{I}_{n}=\mathcal{I} \cap \mathcal{R}_{n}$. Then if $\mathcal{S}_{n}=\mathcal{R}_{n} / \mathcal{I}_{n}, \mathcal{S}$ is the direct sum of the $\mathcal{S}_{n}$ and $H_{n}^{\mathcal{S}}=\operatorname{dim} \mathcal{S}_{n}$, the number of independent homogeneous functions of degree $n$ modulo the $h_{m}$, which is the number of independent functions we have to consider. These dimensions are encoded into a formal series, the Hilbert series (see, e.g., [42]),

$$
H^{\mathcal{S}}(t)=\sum H_{n}^{\mathcal{S}} t^{n}
$$

If $\mathcal{J}$ is a homogeneous ideal in $\mathcal{R}, \mathcal{T}=\mathcal{R} / \mathcal{J}$ and $h \in \mathcal{R}_{s}$, a homogeneous polynomial of degree $s, h \notin \mathcal{J}$, we can consider the ideal $\mathcal{K}$, generated by $\mathcal{J}$ and $h$, i.e. $\mathcal{K}=\left\{g_{1}+h g_{2}\right.$ : $\left.g_{1} \in \mathcal{J}, g_{2} \in \mathcal{R}\right\}$, and the corresponding quotient ring, $\mathcal{U}=\mathcal{R} / \mathcal{K}$. Define $\phi: \mathcal{T} \rightarrow \mathcal{T}$ by $\phi(g+\mathcal{J})=g h+\mathcal{J}$. The kernel of $\phi$ consists of those $(g+\mathcal{J}) \in \mathcal{T}$, for which $g h \in \mathcal{J}$, so $\operatorname{ker} \phi$ is trivial if $g h \notin \mathcal{J}$ whenever $g \notin \mathcal{J}$. If there is $g \notin \mathcal{J}$ with $g h \in \mathcal{J}, h$ is said to be a zero divisor in $\mathcal{T}$. If $h$ is a nonzero divisor in $\mathcal{T}, \operatorname{ker} \phi$ is trivial, and it is easy to calculate the Hilbert series of $\mathcal{U}$ in terms of that of $\mathcal{T}$.

Because $\mathcal{J} \subset \mathcal{K}$, we can define $\psi: \mathcal{T} \rightarrow \mathcal{U}$ by $\psi(g+\mathcal{J})=g+\mathcal{K}$. The kernel of $\psi$ consists of $(g+\mathcal{J}) \in \mathcal{T}$ such that $g \in \mathcal{K}$, implying $g+\mathcal{J}=g^{\prime} h+\mathcal{J}$, for some $g^{\prime}$, implying $\operatorname{ker} \psi=\operatorname{im} \phi$, while, clearly, $\operatorname{im} \psi=\mathcal{U}$. If $\operatorname{deg} h=d$, we have maps $\phi: \mathcal{T}_{n} \rightarrow \mathcal{T}_{n+d}$ and $\psi: \mathcal{T}_{n} \rightarrow \mathcal{U}_{n}$. So, provided that $h$ is a nonzero divisor in $\mathcal{T}$, we have an exact sequence:

$$
0 \longrightarrow \mathcal{T}_{n} \stackrel{\phi}{\longrightarrow} \mathcal{T}_{n+d} \stackrel{\psi}{\longrightarrow} \mathcal{U}_{n+d} \longrightarrow 0
$$

$\operatorname{dim} \mathcal{T}_{n}=\operatorname{dim}[\operatorname{im} \phi]_{n+d}, \operatorname{dim} \mathcal{T}_{n+d}=\operatorname{dim}[\operatorname{ker} \psi]_{n+d}+\operatorname{dim}[\operatorname{im} \psi]_{n+d}=\operatorname{dim} \mathcal{T}_{n}+\operatorname{dim} \mathcal{U}_{n+d}$, using $\operatorname{im} \phi=\operatorname{ker} \psi$. Multiplying by $t^{n+d}$ and summing gives

$$
H^{\mathcal{U}}(t)=\left(1-t^{d}\right) H^{\mathcal{T}}(t)
$$

provided that $h$ is a non-zero divisor in $\mathcal{T}$. 
We can use this result to calculate $H^{\mathcal{S}}(t)$ iteratively if $h_{m}$ is a nonzero divisor in $\mathcal{R} / \mathcal{I}^{(m-1)}, 2 \leq m \leq N-3$, where $\mathcal{I}^{(m)}$ denotes the ideal $\left\langle h_{1}, \ldots, h_{m}\right\rangle$, generated by $h_{1}, \ldots, h_{m}$. In this case, $h_{1}, \ldots, h_{N-3}$ is said to be a regular sequence, and we can apply (5.8) iteratively to obtain

$$
H^{\mathcal{S}^{(m)}}(t)=\left(1-t^{m}\right) H^{\mathcal{S}^{(m-1)}}(t), \quad 1 \leq m \leq N-3,
$$

where $\mathcal{S}^{(m)}=\mathcal{R} / \mathcal{I}^{(m)}, \mathcal{S}^{(0)}=\mathcal{R}$. So, since $H^{\mathcal{R}}(t)=(1-t)^{2-N}$,

$$
H^{\mathcal{S}}(t)=\frac{1}{(1-t)^{N-2}} \prod_{m=1}^{N-3}\left(1-t^{m}\right)=\frac{1}{1-t} \prod_{m=2}^{N-3}\left(1+t+\ldots+t^{m-1}\right)
$$

consistent with results obtained by computer calculation in [11] for low values of $N$.

That, in the case of the scattering equations, $h_{1}, \ldots, h_{N-3}$ is indeed a regular sequence follows from the Unmixedness Theorem of Macaulay [34]. (For an exposition in modern terminology, see, e.g., [43], specially section 5.) This theorem states that, if $g_{1}, g_{2}, \ldots g_{s}$ is a regular sequence of homogeneous polynomials, in the $M$ (complex) variables, $x_{1}, x_{2}, \ldots, x_{M}$, then the (projective) variety defined by $g_{1}=g_{2}=\cdots=g_{s}=0$ has dimension $M-s$ (i.e. projective dimension $M-s-1$ ), and, conversely, if this variety has dimension $M-s$ then $g_{1}, g_{2}, \ldots g_{s}$ is a regular sequence (irrespective of the order). Taking $M=N-2$, with $x_{a}=z_{a+1}, 1 \leq a \leq N-2$, we have established in section 4 that the variety defined by the scattering equations (5.1) is discrete, i.e. has projective dimension zero, so that, by the Unmixedness Theorem, $h_{1}, h_{2}, \ldots, h_{N-3}$ is a regular sequence.

In fact, we do not have rely on the analysis of section 4 to show that we have a regular sequence. If we supplement the $N-3$ scattering equations (5.1) with the equation $z_{N-1}=0$, there is no solution to this augmented system, other than the trivial solution in which all the $z_{a}$ vanish, because we have already specialized to $z_{N}=0$, and we established in [10] that the values of the $z_{a}$ must be distinct for different $a$ if the Mandelstam variables $\sigma_{S}=k_{S_{1}}^{2}$ are nonzero. [We can also deduce this directly by first substituting $z_{N-1}=0$ in $h_{N-3}=0$ to obtain

$$
\sigma_{A^{\prime \prime}} z_{2} z_{3} \cdots z_{N-2}=0,
$$

where $A^{\prime \prime}=\{a \in A: a \neq 1, N-1, N\}$, implying that one of $z_{a}, 2 \leq a \leq N-2$, vanishes, $z_{b}$ say. Then, putting $z_{b}=z_{N-1}=0$ in $h_{N-2}=0$, we can deduce that another $z_{a}$ vanishes and so on to establish that all the $z_{a}, 2 \leq a \leq N-1$, vanish, showing again that there is no nontrivial solution to (5.1) together with $z_{N-1}=0$.] So the space of solutions has (affine) dimension zero, i.e. is empty projectively, and by the Unmixedness Theorem, $h_{1}, h_{2}, \ldots, h_{N-3}, z_{N-1}$ is a regular sequence, and it follows that the subsequence $h_{1}, h_{2}, \ldots, h_{N-3}$ is regular.

[A somewhat similar argument has been used in [44] to show that the (nonhomogeneous) polynomials obtained by putting $z_{2}=1$ in $h_{1}, \ldots, h_{N-3}$ form a H-basis, in the sense of Macauley, for the (affine) ideal that they generate.]

The regularity of the sequence of scattering equations implies that the formula (5.10) for the Hilbert series holds. $H_{n}^{\mathcal{S}}$, as a function of $n$, is called the Hilbert function. For sufficiently large $n$, it is always a polynomial whose degree equals the projective dimension 
of the variety, and so is eventually constant for a zero-dimensional variety, with the constant equalling the number of points in the discrete variety, counted according to multiplicity. In this case, $H_{n}^{\mathcal{S}}=(N-3)$ ! for $n \geq \frac{1}{2}(N-3)(N-4)$, corresponding to the fact that the scattering equations have $(N-3)$ ! solutions.

The techniques of this subsection can be applied to the generalizations of the scattering equations, corresponding to Möbius spin $\frac{1}{2} N-\ell, 2 \leq \ell \leq \frac{1}{2} N$, introduced in [10]. (The values $\ell=0,1$ correspond to overdetermined systems.) These are systems of $N-2 \ell+1$ homogeneous equations, in $N-2$ variables, $z_{a}$, of each degree from $\ell-1$ to $N-\ell-1$. ( $\ell=2$ corresponds to polynomial form of the original CHY scattering equations.) These equation are linear in each of the $z_{a}$. Provided that the coefficients in the polynomials are all nonzero, after supplementing the $N-2 \ell+1$ homogeneous polynomials by $2 \ell-1$ of the $z_{a}$, we can use a argument as in (5.11) to show that this system of $N-2$ equations in $N-2$ variables has only a trivial solution. So, again, the polynomials form a regular sequence and the Hilbert series for the associated ring, $\mathcal{S}=\mathcal{R} / \mathcal{I}$, where $\mathcal{I}$ is the ideal generated by the $N-2 \ell+1$ homogeneous polynomials is given by

$$
H^{\mathcal{S}}(t)=\frac{1}{(1-t)^{N-2}} \prod_{m=\ell-1}^{N-\ell-1}\left(1-t^{m}\right)=\frac{1}{(1-t)^{2 \ell-3}} \prod_{m=\ell-1}^{N-\ell-1}\left(1+t+\ldots+t^{m-1}\right)
$$

so that the $H_{n}^{\mathcal{S}}$ is a polynomial of degree $2 \ell-4$ for sufficiently large $n$, corresponding to a variety of this projective dimension. This expression for $H^{\mathcal{S}}(t)$ is again consistent with results in [11] for low values of $N$.

\section{Acknowledgments}

LD was partially supported by NSF grant No. PHY-1620311, and thanks the Institute for Advanced Study at Princeton for its hospitality. PG was partially supported by NSF grant No. PHY-1314311.

Open Access. This article is distributed under the terms of the Creative Commons Attribution License (CC-BY 4.0), which permits any use, distribution and reproduction in any medium, provided the original author(s) and source are credited.

\section{References}

[1] D.B. Fairlie and D.E. Roberts, Dual Models without Tachyons - a New Approach, unpublished Durham preprint PRINT-72-2440 (1972).

[2] D.E. Roberts, Mathematical Structure of Dual Amplitudes, Ph.D. Thesis, Durham University, Durham U.K. (1972), available at Durham E-Theses online: http://etheses.dur.ac.uk/8662/1/8662_5593.PDF.

[3] D.B. Fairlie, A Coding of Real Null Four-Momenta into World-Sheet Co-ordinates, Adv. Math. Phys. 2009 (2009) 284689 [arXiv:0805.2263] [InSPIRE].

[4] D.J. Gross and P.F. Mende, String Theory Beyond the Planck Scale, Nucl. Phys. B 303 (1988) 407 [InSPIRE]. 
[5] F. Cachazo, S. He and E.Y. Yuan, Scattering equations and Kawai-Lewellen-Tye orthogonality, Phys. Rev. D 90 (2014) 065001 [arXiv:1306.6575] [INSPIRE].

[6] F. Cachazo, S. He and E.Y. Yuan, Scattering of Massless Particles in Arbitrary Dimensions, Phys. Rev. Lett. 113 (2014) 171601 [arXiv:1307.2199] [InSPIRE].

[7] F. Cachazo, S. He and E.Y. Yuan, Scattering of Massless Particles: Scalars, Gluons and Gravitons, JHEP 07 (2014) 033 [arXiv:1309.0885] [INSPIRE].

[8] F. Cachazo, S. He and E.Y. Yuan, Scattering Equations and Matrices: From Einstein To Yang-Mills, DBI and NLSM, JHEP 07 (2015) 149 [arXiv:1412.3479] [INSPIRE].

[9] L. Dolan and P. Goddard, Proof of the Formula of Cachazo, He and Yuan for Yang-Mills Tree Amplitudes in Arbitrary Dimension, JHEP 05 (2014) 010 [arXiv:1311.5200] [INSPIRE].

[10] L. Dolan and P. Goddard, The Polynomial Form of the Scattering Equations, JHEP 07 (2014) 029 [arXiv: 1402.7374] [INSPIRE].

[11] Y.-H. He, C. Matti and C. Sun, The Scattering Variety, JHEP 10 (2014) 135 [arXiv: 1403.6833] [INSPIRE].

[12] R. Huang, J. Rao, B. Feng and Y.-H. He, An Algebraic Approach to the Scattering Equations, JHEP 12 (2015) 056 [arXiv: 1509.04483] [INSPIRE].

[13] M. Søgaard and Y. Zhang, Scattering Equations and Global Duality of Residues, Phys. Rev. D 93 (2016) 105009 [arXiv:1509.08897] [InSPIRE].

[14] C. Cardona and C. Kalousios, Comments on the evaluation of massless scattering, JHEP 01 (2016) 178 [arXiv:1509.08908] [INSPIRE].

[15] S.G. Naculich, Scattering equations and BCJ relations for gauge and gravitational amplitudes with massive scalar particles, JHEP 09 (2014) 029 [arXiv:1407.7836] [INSPIRE].

[16] L. Mason and D. Skinner, Ambitwistor strings and the scattering equations, JHEP 07 (2014) 048 [arXiv: 1311.2564] [INSPIRE].

[17] T. Adamo, E. Casali and D. Skinner, Ambitwistor strings and the scattering equations at one loop, JHEP 04 (2014) 104 [arXiv: 1312.3828] [INSPIRE].

[18] N.E.J. Bjerrum-Bohr, P.H. Damgaard, P. Tourkine and P. Vanhove, Scattering Equations and String Theory Amplitudes, Phys. Rev. D 90 (2014) 106002 [arXiv:1403.4553] [INSPIRE].

[19] E. Casali and P. Tourkine, Infrared behaviour of the one-loop scattering equations and supergravity integrands, JHEP 04 (2015) 013 [arXiv:1412.3787] [INSPIRE].

[20] L. Dolan and P. Goddard, unpublished.

[21] L. Dolan, The Scattering Equations, their Properties and Proofs, talk at New geometric structures in scattering amplitudes, Oxford University, Oxford U.K. (2014), http://www.maths.ox.ac.uk/events/conferences/past-events/new-geometric-structures -scattering-amplitudes.

[22] P. Goddard, Properties of the Scattering Equations, talk at Current Themes in High Energy Physics and Cosmology, Niels Bohr International Academy, Copenhagen Denmark (2015), https://indico.nbi.ku.dk/conferenceOtherViews.py?view=nicecompact\&confId=751.

[23] Y. Geyer, L. Mason, R. Monteiro and P. Tourkine, Loop Integrands for Scattering Amplitudes from the Riemann Sphere, Phys. Rev. Lett. 115 (2015) 121603 [arXiv:1507.00321] [INSPIRE]. 
[24] C. Baadsgaard, N.E.J. Bjerrum-Bohr, J.L. Bourjaily, S. Caron-Huot, P.H. Damgaard and B. Feng, New Representations of the Perturbative S-matrix, Phys. Rev. Lett. 116 (2016) 061601 [arXiv:1509.02169] [INSPIRE].

[25] C. Baadsgaard, N.E.J. Bjerrum-Bohr, J.L. Bourjaily, P.H. Damgaard and B. Feng, Integration Rules for Loop Scattering Equations, JHEP 11 (2015) 080 [arXiv:1508.03627] [INSPIRE].

[26] Y. Geyer, L. Mason, R. Monteiro and P. Tourkine, One-loop amplitudes on the Riemann sphere, JHEP 03 (2016) 114 [arXiv:1511.06315] [INSPIRE].

[27] S. He and E.Y. Yuan, One-loop Scattering Equations and Amplitudes from Forward Limit, Phys. Rev. D 92 (2015) 105004 [arXiv:1508.06027] [INSPIRE].

[28] J.J. Sylvester, On Derivation of Coexistence, Part II, being the Theory of Simultaneous Homogeneous Equations, Philos. Mag. 15 (1839) 428.

[29] A. Cayley, On the Theory of Elimination, Cambridge Dublin Math. J. 3 (1848) 116.

[30] G. Salmon, Lessons Introductory to the Modern Higher Algebra, BiblioLife Dublin Ireland (1885).

[31] I.M. Gel'fand, M.M. Kapranov and A.V. Zelevinsky, Discriminants, Resultants and Multidimensional Determinants, Birkhäuser, Boston U.S.A. (1994).

[32] B. Sturmfels, CBMS Regional Conference Series in Mathematics. No. 97: Solving Systems of Polynomial Equations, AMS Press, Providence U.S.A. (2002).

[33] M.J. Duff, String triality, black hole entropy and Cayley's hyperdeterminant, Phys. Rev. D 76 (2007) 025017 [hep-th/0601134] [INSPIRE].

[34] F.S. Macaulay, Cambridge Tracts in Mathematics and Mathematical Physics. Vol. 19: The Algebraic Theory of Modular Systems, Cambridge University Press, Cambridge U.K. (1916).

[35] C. Cardona and C. Kalousios, Elimination and recursions in the scattering equations, Phys. Lett. B 756 (2016) 180 [arXiv:1511.05915] [INSPIRE].

[36] F.S. Macaulay, Some Formulae in Elimination, Proc. London Math. Soc. 35 (1902) 3.

[37] J.P. Jouanolou, Le Formalisme du Résultant, Adv. Math. 90 (1991) 117.

[38] D.A. Cox, J. Little and D. O'Shea, Using Algebraic Geometry, Springer-Verlag, New York U.S.A. (2004).

[39] I.M. Gel'fand, M.M. Kapranov and A.V. Zelevinsky, Hyperdeterminants, Adv. Math. 96 (1992) 226.

[40] A. Dickenstein and I.Z. Emiris, Multihomogeneous Resultant Matrices, in Proceedings of the 2002 international symposium on Symbolic and algebraic computation, Lille France (2002), ACM Press, New York U.S.A. (2002).

[41] B. Sturmfels and A. Zelevinsky, Multigraded Resultants of Sylvester Type, J. Algebra 163 (1994) 115.

[42] D. Eisenbud, Graduate Texts in Mathematics. Vol. 150: Commutative Algebra, Springer-Verlag, New York U.S.A. (1995).

[43] H.M. Möller and T. Sauer, H-bases for Polynomial Interpolation and System Solving, Adv. Comput. Math. 12 (2000) 335.

[44] J. Bosma, M. Søgaard and Y. Zhang, The Polynomial Form of the Scattering Equations is an H-Basis, Phys. Rev. D 94 (2016) 041701 [arXiv: 1605.08431] [INSPIRE]. 